\title{
Isolation and Characterization of 1,3-Bis(vinylbenzyl)thymine: Copolymerization with Vinylbenzyl Thymine Ammonium Chloride
}

\author{
Ngoc Chau H. Vy, ${ }^{1}$ Nina Bin Chen, ${ }^{1}$ Debora M. Martino, ${ }^{2}$ John C. Warner, ${ }^{2}$ and Nancy Lee ${ }^{1}$ \\ ${ }^{1}$ Simmons College, Department of Chemistry, 300 The Fenway, Boston, MA 02215, USA \\ ${ }^{2}$ Warner Babcock Institute for Green Chemistry, 100 Research Dr., Wilmington, MA 01887, USA \\ Correspondence should be addressed to Debora M. Martino; debora.martino@warnerbabcock.com \\ and Nancy Lee; nancy.lee@simmons.edu
}

Received 10 September 2016; Accepted 22 November 2016; Published 4 January 2017

Academic Editor: Dirk Kuckling

Copyright (c) 2017 Ngoc Chau H. Vy et al. This is an open access article distributed under the Creative Commons Attribution License, which permits unrestricted use, distribution, and reproduction in any medium, provided the original work is properly cited.

\begin{abstract}
A novel bioinspired molecule, 1,3-bis(vinylbenzyl)thymine (bisVBT), was isolated as a by-product during the synthesis of 1-(4vinylbenzyl)thymine (VBT) and analyzed with various techniques: NMR, IR, and Single-Crystal X-ray Diffraction. In addition to embodying all the desired characteristics of VBT (i.e., having the ability to undergo a $2 \pi+2 \pi$ photodimerization reaction upon UV irradiation, a derivatization site, hydrogen bonding sites, and aromatic stacking ability) the bisVBT monomer has the added benefit of having two vinyl groups for cross-polymerization. Copolymerizing the bisVBT monomer with the charged monomer vinylbenzyl triethylammonium (VBA) chloride, different copolymers/terpolymers/cross-linked network were obtained, as it was shown by the absence of the vinyl resonance in the NMR spectra. Thermal Gravimetric Analysis (TGA) and Differential Scanning Calorimetry (DSC) showed an indication of materials with low degree of cross-linking. A Gel Permeation Chromatography (GPC) method was improved to better characterize the molecular weight distributions of the cationic structures. Preliminary qualitative cross-linking studies were performed on bisVBT-VBA copolymers, and a comparison with VBT-VBA copolymers is presented.
\end{abstract}

\section{Introduction}

The study of bioinspired environmentally benign, nontoxic, and recyclable thymine-based polymers has been on a steady rise in the past decades, due to their photosensitivity and molecular recognition patterns [1-4]. A specific example of such a molecule is 1-(4-vinylbenzyl)thymine (VBT) $[5,6]$.

In designing VBT copolymers, one of the desirable features that previous researchers incorporated into the copolymer is the ability to be processed in aqueous systems. Due to the high level of intermolecular forces between each monomer, the VBT homopolymer is insoluble in water. Thus, VBT is often polymerized in different ratios with styrene derivative charged monomers, such as anionic vinyl phenyl sulfonate (VPS) salts [7] or cationic vinylbenzyl triethylammonium chloride (VBA) [8-10] which allows the copolymer to be soluble in water. Extending the knowledge achieved from the study of the natural phenomena related to thymine reactivity and intermolecular interactions has generated a diapason of potential applications [11-14] ranging from photoresist material $[15,16]$ and organic-inorganic hybrid devices [2] to antibacterial coatings [17], drug delivery systems [3,18-20], recyclable plastics [21], and biosensors $[22,23]$. On the other hand, the increasing attention given to the environmental and toxicological dilemmas linked to commercial materials situates the thymine-based polymeric systems as a Green Chemistry platform [24]. From an intellectual standpoint, thymine polymeric systems offer an approach to physiologically relevant processes, while offering commercially applicable opportunities in material sciences. This synergy will make available new comprehensions and the development of innovative materials.

Quaternary ammonium groups, such as in VBA, are known to exhibit antimicrobial properties as they interact with the cell walls of certain types of bacteria and cause the destruction of cells by lysins. This property in 
combination with the photo-induced immobilization of VBT monomers creates a host of possible applications for VBTVBA copolymers, such as antimicrobial coatings in hospitals or antifouling agents on the exterior of boats and ships. Furthermore, the ethyl alkyl arms on VBA can be replaced with alkyl groups of different chain lengths to vary the antimicrobial properties and applications [17]. To ensure that the presence of charged monomers would not interfere with the photo-cross-linking reaction of VBT moieties, irradiation studies have been performed on varying ratios of VBT-VBA copolymers to quantify the extent of their photodimerization and immobilization $[8,10]$. From such studies, it was found that linear VBT-VBA copolymer chains cross-link through adjacent thymine moieties to form an immobilized thin film.

Inspired by these findings and in the attempt to incorporate Green Chemistry [24] in our practices, we focused our research on improving the synthesis of the VBT monomer by isolating and characterizing each product/by-product. One of the most common routes of the VBT synthesis is via a nucleophilic substitution reaction between vinylbenzyl chloride (VBC) and potassium thyminate (KThy) [5]. Although this method of synthesis has been predominantly used to produce VBT, its yield has not been known to exceed $40 \%$. Our meticulous studies permitted obtaining a high yield of VBT monomer (37.4\%) and correspondingly other reaction byproducts such as 1,3-bis(4-vinylbenzyl)thymine (bisVBT) $(22.1 \%)$ and trace amounts of 3-(4-vinylbenzyl)thymine (3VBT) and 4-vinylbenzyl alcohol (VBOH). Out of these byproducts, bisVBT occurred in the highest quantity, making it the easiest to isolate and characterize.

Due to the presence of only one vinyl group in its structure, the VBT monomer is only able to form linear copolymers. However, since the bisVBT monomer has two vinyl groups, it has the potential to form branched and even cross-linked networks. With the bisVBT enhanced polymerizability, it can be anticipated that the strength and flexibility of cross-linked bisVBT materials will meet the requirements of new desired applications. Furthermore, the bisVBT monomer similarly has photo-cross-linking capabilities due to the thymine base in its structure. This work describes the synthesis of three different bisVBT-VBA materials of varying molecular weights and structure and a comparison of their thermal profiles and cross-linking efficiencies to previously synthesized VBT-VBA copolymers.

A Gel Permeation Chromatography (GPC) method was modified to better characterize the molecular weight distributions of the synthesized bisVBT-VBA cationic networks. Preliminary qualitative cross-linking studies were performed on bisVBT-VBA materials and a comparison with wellknown VBT-VBA copolymers is presented.

\section{Experimental Section}

2.1. Materials. Thymine (99\%), vinylbenzyl chloride (VBC) (90\%), and butylated hydroxytoluene (BHT) (99\%) were purchased from Sigma-Aldrich. Sodium hydroxide (99\%) was purchased from Fisher Scientific. Ethanol (95\%), isopropanol (99\%, ACS grade), and acetone (99.9\%) were purchased from Pharmco-Aaper. Dimethyl formamide (DMF, 99.8\%) was purchased from VWR. Ethyl acetate (ACS grade) and hexanes (ACS grade) were purchased from Macron. Water (HPLC grade) was purchased from Spectrum. Deuterated DMSO (99.9\%) and deuterated chloroform $\left(\mathrm{CDCl}_{3}, 99.9 \%\right)$ were purchased from Cambridge Isotope Laboratories. Poly(2vinylpyridine) polymer standards were purchased from Jordi Labs. All reagents were used as received unless mentioned otherwise.

PET film (Melinex 454/500) was provided by DuPont Teijin Films. Film coating was performed using a microscope slide edge to draw down the copolymer solution. Irradiation of the copolymers films was carried out using Spectronics Corp. Spectrolinker XL-1500 (254 nm, 40 W).

All synthesized monomers and copolymers were dried in a vacuum oven $\left(\mathrm{VWR}^{\circledR} 1410 \mathrm{~T}=80^{\circ} \mathrm{C}\right.$; pressure $=$ $28 \mathrm{inHg}$ ). Purification of VBT and bisVBT were performed using Teledyne Isco CombiFlash Rf 200. ${ }^{1} \mathrm{H}$ NMR spectra of monomers and copolymers were performed using a $90 \mathrm{MHz}$ (Anasazi EFT) and a $400 \mathrm{MHz}$ (JEOL) NMR spectrometers. IR analysis was performed using Thermo Scientific Nicolet 6700 FTIR instrument. Agilent 8453 Diode Array UVVis Spectrophotometer was used for sample measurement. Molecular weights were determined by GPC performed using a Beckman Gold Programmable Solvent Module HPLC system (126) with a UV/Vis Programmable Detector Module (166). A guard column and two analytical columns (Jordi DVB Polar Pack Wax mixed bed) were used. Copolymer solutions in the solvent were agitated on an orbital shaker overnight (recommended by the GPC-columns manufacturer) and filtered through a $0.45 \mu \mathrm{m}$ Whatman filter attached to a BD $1 \mathrm{~mL}$ Luer-Lok tip syringe before sample injection. GPC mobile phase filtration apparatus was purchased from Quark Glass.

Thermal studies of the copolymers were performed as follows. TGA data were collected using a TA Instrument Q5000 TG Analyzer. In a typical TGA experiment, the copolymer sample was removed from the vacuum oven, carefully weighed, put into the furnace of the instrument, and heated, under nitrogen, over a range of $20^{\circ} \mathrm{C}-600^{\circ} \mathrm{C}$ on a ramp of $20^{\circ} \mathrm{C} / \mathrm{min}$. DSC data were collected using a TA Instruments Q2000 DS Calorimeter. Samples for the DSC experiment were carefully weighed, heated in Tzero aluminum hermetic pans purchased from TA Instruments. Heat-cool-heat DSC experiments were performed under $\mathrm{N}_{2}$ flux, a heating ramp of $10^{\circ} \mathrm{C} / \mathrm{min}$, and a cooling ramp of $5^{\circ} \mathrm{C} / \mathrm{min}$.

2.2. Synthesis of Sodium Thyminate. Sodium hydroxide $(2.04 \mathrm{~g}, 0.051 \mathrm{~mol})$ was added to a flask containing DI water $(30 \mathrm{~mL})$, heated to $50^{\circ} \mathrm{C}$, and stirred at $300 \mathrm{RPM}$ until dissolved. Then thymine $(6.50 \mathrm{~g}, 0.044 \mathrm{~mol})$ was added and stirred for additional 20 minutes. After the solution has completely turned clear, the flask was removed from heat and cooled to room temperature. $50 \mathrm{~mL}$ of ethanol was added slowly to the stirring solution and a white precipitate was observed. The white solid was filtered with a Buchner funnel, rinsed with additional ethanol, and dried overnight in a vacuum oven at $80^{\circ} \mathrm{C}$ under 25 in $\mathrm{Hg}$ vacuum. Sodium 


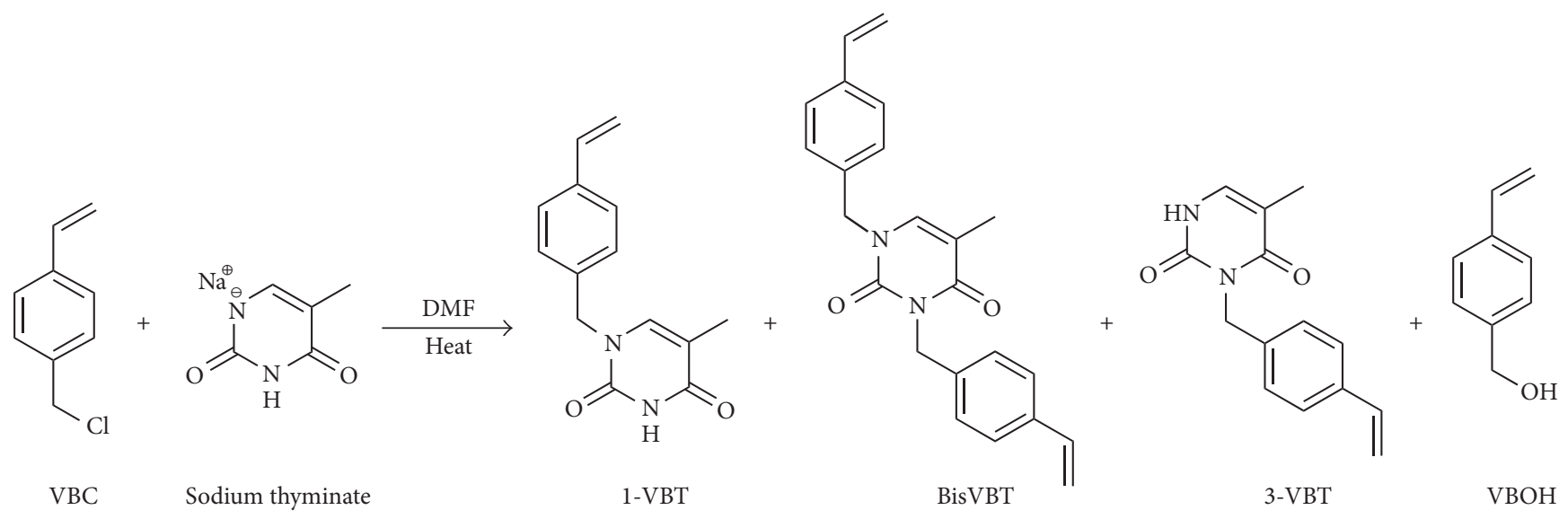

FIGURE 1: Reaction scheme for the synthesis of 1-(4-vinylbenzyl)thymine (VBT) and byproducts, 1,3-bis(4-vinylbenzyl)thymine (bisVBT), 3-(4-vinylbenzyl)thymine (3-VBT), and 4-vinylbenzyl alcohol (VBOH) in order of decreasing relative amounts.

thyminate collected $(3.80 \mathrm{~g}, 49.85 \%$ yield) was characterized by ${ }^{1} \mathrm{H}$ NMR and IR.

${ }^{1} \mathrm{H}$ NMR $(90 \mathrm{MHz}$, DMSO-d 6 , $\delta$, ppm): $7.44(\mathrm{~s}, 1 \mathrm{H}, \mathrm{C}=\mathrm{C}-$ $\mathrm{H}), 1.69(\mathrm{~s}, 3 \mathrm{H})$.

IR $\left(\mathrm{KBr}\right.$ pellet, $\left.\mathrm{cm}^{-1}\right): 3664,3331,2560,2198,1967,1722$, $1689,1629,1490,1467,1384,1373,1337,1291,1215,1020,959$, $947,863,787,754,676,621,592,487,447$.

2.3. Synthesis and Purification of Monomers, VBT, and bisVBT. VBT synthesis was slightly modified from procedures described previously $[5,6]$. The reaction scheme is presented in Figure 1.

In a $250 \mathrm{~mL}$ round bottom flask containing a magnetic stirring bar, dimethylformamide (DMF) $(20 \mathrm{~mL})$ was added and heated in an oil bath until the temperature was stabilized at $70^{\circ} \mathrm{C}$. To the same flask, sodium thyminate (NaThy, $3.5 \mathrm{~g}, 0.024 \mathrm{~mol}$ ) was added. Once a majority of NaThy was dissolved to give a white milky solution, vinylbenzyl chloride (VBC) $(4.8 \mathrm{~g}, 0.028 \mathrm{~mol})$ and inhibitor 2,6-di-tertbutyl-4methylphenol (BHT) $(5 \mathrm{mg}, 0.02 \mathrm{mmol})$ were added. The light yellow opaque mixture was allowed to heat at $70^{\circ} \mathrm{C}$ and stir for 24 hours. The DMF solvent was removed at the end of the reaction via rotary evaporation under vacuum. The paste-like residue was redissolved in methylene chloride and adsorbed onto silica gel. RediSep Rf prepacked silicagel columns (silica $80 \mathrm{~g}$ ) were used to separate the reaction mixture on a Teledyne Isco CombiFlash instrument. The elution solvent system used was a gradual ramp from $20 \%$ ethyl acetate in hexanes to $50 \%$ at $60 \mathrm{~mL} / \mathrm{min}$, with a total separation time of 60 minutes. The isolated VBT $(2.14 \mathrm{~g}$, $37.4 \%$ yield) was a white solid. The isolated bisVBT $(1.87 \mathrm{~g}$, $22.1 \%$ yield) was a translucent white viscous liquid, which solidified into an opaque white solid upon cooling to $4^{\circ} \mathrm{C}$. Both of the isolated VBT and bisVBT products were placed in a vacuum oven to dry and then characterized using NMR (JEOL $400 \mathrm{MHz}$ ) and IR. Based on ${ }^{1} \mathrm{H}$ NMR spectra results, the monomers were deemed pure enough for the synthesis of the copolymers.

To examine the crystal packing behavior of the synthesized bisVBT monomer, crystalline samples were subjected to crystal and molecular structure determinations by SingleCrystal X-ray Diffraction. The data were collected using an APEXII BRUKER X-ray diffractometer with X-ray radiation generated from a Mo sealed X-ray tube $\left(K_{\alpha}=0.70173 \AA\right.$ with a potential of $40 \mathrm{kV}$ and a current of $40 \mathrm{~mA}$ ) at the Department of Chemistry, Texas A\&M University. Detailed X-ray diffraction data are available in the Electronic Supplementary Information.

\subsubsection{1-(4-Vinylbenzyl)thymine Monomer}

${ }^{1} \mathrm{HNMR}\left(400 \mathrm{MHz}, \mathrm{CDCl}_{3}, \delta\right.$, ppm): 1.87 (s, 3H), $4.87(\mathrm{~s}, 2 \mathrm{H}), 5.27(\mathrm{~d}, J=10.28 \mathrm{~Hz}, 1 \mathrm{H}), 5.76(\mathrm{~d}$, $J=17.60 \mathrm{~Hz}, 1 \mathrm{H}), 6.81\left(\mathrm{dd}, J_{1}=18.52, J_{2}=10.76\right.$, $1 \mathrm{H}), 6.96(\mathrm{~s}, 1 \mathrm{H}), 7.24(\mathrm{~d}, J=7.32 \mathrm{~Hz}, 2 \mathrm{H}), 7.41(\mathrm{~d}$, $J=8.32 \mathrm{~Hz}, 2 \mathrm{H}), 8.45(\mathrm{~s}, 1 \mathrm{H})$.

\subsubsection{1,3-Bis(4-vinylbenzyl)thymine Monomer}

${ }^{1} \mathrm{HNMR}\left(400 \mathrm{MHz}, \mathrm{CDCl}_{3}, \delta, \mathrm{ppm}\right): 1.57\left(\mathrm{H}_{2} \mathrm{O}\right), 1.89$ $(\mathrm{s}, 3 \mathrm{H}), 4.88(\mathrm{~s}, 2 \mathrm{H}), 5.14(\mathrm{~s}, 2 \mathrm{H}), 5.23(\mathrm{~d}, J=11.89 \mathrm{~Hz}$, $1 \mathrm{H}), 5.29(\mathrm{~d}, J=12.02 \mathrm{~Hz}, 1 \mathrm{H}), 5.73(\mathrm{~d}, J=16.03 \mathrm{~Hz}$, $1 \mathrm{H}), 5.76(\mathrm{~d}, J=16.12 \mathrm{~Hz}, 1 \mathrm{H}), 6.66\left(\mathrm{dd}, J_{1}=10.72\right.$, $\left.J_{2}=6.60,1 \mathrm{H}\right), 6.81\left(\mathrm{dd}, J_{1}=11.17, J_{2}=7.45,1 \mathrm{H}\right)$, $6.95(\mathrm{~s}, 1 \mathrm{H}), 7.26(\mathrm{~d}, J=7.43 \mathrm{~Hz}, 2 \mathrm{H}), 7.35(\mathrm{~d}, J=$ $7.84 \mathrm{~Hz}, 2 \mathrm{H}), 7.39(\mathrm{~d}, J=7.53 \mathrm{~Hz}, 2 \mathrm{H}), 7.45(\mathrm{~d}, J=$ $7.44 \mathrm{~Hz}, 2 \mathrm{H})$.

${ }^{13} \mathrm{C}$ NMR $\left(400 \mathrm{MHz}, \mathrm{CDCl}_{3}, \delta, \mathrm{ppm}\right): 163.5,151.7$, $137.8,137.6,136.8,136.5,136.4,136.0,134.9,129.3,128.1$, $126.8,126.2,114.64,113.8,110.4,51.6,44.4,13.1$.

IR $\left(\mathrm{cm}^{-1}\right): 1694,1661,1627,1461,1338,1212,992,896$, $865,826,778,760$.

\subsection{Synthesis of bisVBT-VBA Copolymers}

2.4.1. Synthesis of 1:32 bisVBT-VBA Copolymer (1\% AIBN). Vinylbenzyl triethylammonium chloride (VBA) monomer was synthesized as described before [25]. In a threeneck $500 \mathrm{~mL}$ round bottom flask equipped with a stirring 
bar, reflux condenser, and nitrogen inlet, bisVBT (0.50 g, $1.4 \mathrm{mmol}, 1 \mathrm{~mol} \mathrm{eq}$.) was dissolved in isopropanol $(115 \mathrm{~mL})$ at $80^{\circ} \mathrm{C}$. Upon complete dissolution of bisVBT, the reaction temperature was brought down to $65^{\circ} \mathrm{C}$. At this point VBA (11.31 g, $44.6 \mathrm{mmol}, 32 \mathrm{~mol} \mathrm{eq.)} \mathrm{was} \mathrm{added} \mathrm{swiftly} \mathrm{to} \mathrm{min-}$ imize water absorption. Following complete dissolution of VBA, 2,2' -azobisisobutyronitrile (AIBN, $0.125 \mathrm{~g}, 1 \% \mathrm{w} / \mathrm{w}$ ) was added to the reaction mixture. The solution was allowed to stir $(500 \mathrm{rpm})$ at $65^{\circ} \mathrm{C}$ for 24 hours under $\mathrm{N}_{2}$. The solution became notably more viscous as the reaction progressed. At the end of the 24-hour period, the reaction mixture was highly viscous, resembling a clear gel. The copolymer was isolated from the reaction mixture via precipitation in acetone. The mixture was vacuum filtered using Whatman grade 2 filter paper to collect the white copolymer precipitate. The collected copolymer was transferred to an empty beaker and placed in a vacuum oven to dry overnight to give $10.9 \mathrm{~g}$ of copolymer ( $92 \%$ yield). Primary analysis of the copolymer was performed using TLC (90/10 $\mathrm{CHCl}_{3}-\mathrm{MeOH}$ with $1 \%$ $\mathrm{NH}_{4} \mathrm{OH}$ mobile phase) to check for presence of monomer. Characterization of the dried copolymer was carried out using ${ }^{1} \mathrm{H}$ NMR spectroscopy in DMSO- $\mathrm{d}_{6}$, to verify the absence of unreacted monomers and the typical vinyl group signal at chemical shifts between 5 and $6 \mathrm{ppm}$ was not observed in the spectra indicating the formation of a network structure. It should be noted that the copolymer swelled into a highly viscous gel when DMSO was added.

2.4.2. Synthesis of 1:32 bisVBT-VBA Copolymer ( $3 \%$ AIBN). The copolymerization procedure described above was repeated with the following modifications. The weight ratio of AIBN was increased to $3 \%(0.325 \mathrm{~g})$. An overhead stirrer (400 rpm) was used instead of a magnetic stir bar to improve the uniformity of the reaction mixture. The bisVBT solids were ground up prior to being added in isopropanol to improve solubility.

The product of this polymerization reaction was also very viscous and gel-like, similar to the previous reaction. One major difference in appearance was the presence of bubbles in the swollen product. The same acetone vortex was set up to crash out the copolymer. Vacuum filtration with grade 2 filter paper was used to isolate the copolymer particles from the mixture. The isolated white copolymer after drying under vacuum (9.02 g, 76\% yield) appeared to be stickier and more hygroscopic than the previous copolymer.

Preliminary analysis of the copolymer was performed using TLC (90/10 $\mathrm{CHCl}_{3}-\mathrm{MeOH}$ with $1 \% \mathrm{NH}_{4} \mathrm{OH}$ mobile phase) to check for presence of monomer. Characterization of the dried copolymer was performed using ${ }^{1} \mathrm{HNMR}$ spectroscopy with DMSO- $\mathrm{d}_{6}$, to verify the absence of unreacted monomers and the typical vinyl group signal at chemical shifts between 5 and 6 ppm was not observed in the spectra, pointing to a network structure. This copolymer also swelled when DMSO was added but exhibited a lower observed viscosity than the 1:32 bisVBT-VBA ( $1 \% \mathrm{AIBN})$ copolymer.

2.4.3. Synthesis of $1: 4: 32$ bisVBT-VBT-VBA Terpolymer (1\% $A I B N)$. The polymerization procedure described in previous sections was repeated with the following modifications. VBT ( $1.35 \mathrm{~g}, 5.6 \mathrm{mmol}, 4 \mathrm{~mol} \mathrm{eq.)} \mathrm{was} \mathrm{dissolved} \mathrm{in} \mathrm{isopropanol}$ $(115 \mathrm{~mL})$ at $80^{\circ} \mathrm{C}$. Following the complete dissolution of VBT, the temperature was decreased to $65^{\circ} \mathrm{C}$, and bisVBT $(0.50 \mathrm{~g}$, $1.4 \mathrm{mmol}, 1 \mathrm{~mol} \mathrm{eq}$ ) was added to the reaction flask allowing it to dissolve for 15-20 min. VBA (11.3 g, $44.6 \mathrm{mmol}, 32 \mathrm{~mol} \mathrm{eq})$ was added to the reaction mixture. Once all the monomers were dissolved, AIBN (0.130 g, 1\% w/w) was added to initiate polymerization. An overhead stirrer (400 rpm) was used [26].

Observation of the reaction flask 18 hours later revealed that while the appearance of the flask contents was clear and gel-like; the stirring action of the overhead stirrer was sluggish, a clear indication that viscosity of this polymerization reaction was higher than in the $1: 32$ bisVBT-VBA copolymer reactions. The stirring rate was turned up to $550 \mathrm{rpm}$ and an additional $100 \mathrm{~mL}$ of isopropanol was added to increase the fluidity of the mixture. After 24 hours, the reaction flask was removed from heat and stirring and the contents were processed similarly with the acetone vortex. While the reaction product was viscous, it still maintained the form of flowing liquid as compared to the gel-like consistency of the 1:32 bisVBT-VBA copolymer reaction products.

A greater volume of acetone was required to be added to the reaction mixture before precipitated particles were observed. While it was possible to see that bisVBT-VBTVBA terpolymer did crash out of solution, the particles were so fine that the suspension resembled skim milk. An initial vacuum filtration was performed using a fritted ceramic funnel with medium pore size, followed by centrifugation for 10 minutes at $5000 \mathrm{rpm}$ and the suspension was allowed to settle by gravity and isolated by decanting. A sludgy layer of sticky white copolymer was observed at the bottom of the beaker, scraped out, and placed in the vacuum oven to dry. The dried copolymer (5.874 g, 45\% yield) was a translucent white hard solid and was analyzed using TLC $\left(90 / 10 \mathrm{CHCl}_{3}\right.$ $\mathrm{MeOH}$ with $1 \% \mathrm{NH}_{4} \mathrm{OH}$ ) to check for presence of monomer. Characterization of the dried copolymer was performed using ${ }^{1}$ HNMR spectroscopy with DMSO- $\mathrm{d}_{6}$, showing the absence of unreacted monomers and confirming a crosslinked network. This terpolymer exhibited slight swelling when dissolved in DMSO and was much less viscous than the previous two copolymers.

2.5. Gel Permeation Chromatography. Molecular weights were determined by GPC experiments performed using Beckman Gold Programmable Solvent Module HPLC system (126) with a UV/Vis Programmable Detector Module (166). A guard column (Jordi DVB Polar Pack Wax mixed bed, $50 \mathrm{~mm} \times 10 \mathrm{~mm}, 5 \mu \mathrm{m}$ particles) and two analytical columns (Jordi DVB Polar Pack Wax mixed bed, $250 \times 10 \mathrm{~mm}$, $5 \mu \mathrm{m}$ particles) in series were used. GPC analyses were performed by comparing the molecular weight of the sample against standards of known molecular weight. Therefore, the choice of the standards used was particularly important for the bisVBT-VBA charged copolymer systems. Poly(2vinylpyridine) standards (PVP) with molecular weights (Mp) of 1,$820 ; 20,900$, and 256,000 were purchased from Jordi Labs. The mobile phase used was 30/70 methanol/water with a total concentration of $1 \mathrm{M}$ acetic acid to maintain the 
positive charge of the column, which required a $\mathrm{pH}$ below 8 . The solvent was made by adding $57.45 \mathrm{~mL}$ of glacial acetic acid to $30 / 70$ methanol (ACS grade) and water (DI) in a $1 \mathrm{~L}$ volumetric flask, followed by vacuum filtration using a mobile phase filtration apparatus (Quark glass) with Nylon 66 membrane $(0.45 \mu \mathrm{m} \times 47 \mathrm{~mm}$, Supelco) . Copolymer sample solutions were prepared by dissolving $2.5 \mathrm{mg}$ of sample per $1 \mathrm{~mL}$ of eluting solvent. The GPC sample solutions were agitated on an orbital shaker overnight at $150 \mathrm{RPM}$ and passed through a $0.45 \mu \mathrm{m}$ Whatman filter attached to a BD $1 \mathrm{~mL}$ Luer-Lok tip syringe before injection. A flow rate of $1.00 \mathrm{~mL} / \mathrm{min}$ was used for all runs at $254 \mathrm{~nm}$ wavelength. Calibration curves were obtained using poly(2-vinylpyridine) standards.

2.6. Irradiation Studies. A $10 \%(\mathrm{w} / \mathrm{w})$ solution of each copolymer was made in 50/50 methanol and water, since it was not possible to dissolve in pure water. Upon addition to the solvent, the bisVBT-VBA (1\% AIBN and 3\% AIBN) copolymers exhibited a significant level of swelling, with the bisVBT-VBA (1\% AIBN) copolymer swelling the most. The clear, colorless solutions had viscosities that resembled a fluid gel. The bisVBT-VBT-VBA terpolymer (1\% AIBN) did not exhibit swelling and was a fluid, clear colorless solution.

The PET substrate was pretreated with $1 \mathrm{M} \mathrm{NaOH}$ for 24 hours in an attempt to etch the surface and improve immobilization of the irradiated copolymer. Copolymer solutions were coated by doctor blading technique onto the PET film (Melinex 454/500) and allowed to dry for $10 \mathrm{~min}$. The PET film was masked and irradiation was set for $30 \mathrm{sec}$, $60 \mathrm{sec}, 120 \mathrm{sec}$, and $180 \mathrm{sec}$. The irradiated coatings were immersed for one minute in FD\&C Blue dye solutions $(0.5 \mathrm{~g}$ of methylene blue dye and $1 \mathrm{~g}$ sodium carbonate in $1 \mathrm{~L}$ of DI water) and then gently dipped in DI water to rinse the extra dye.

\section{Results and Discussion}

The structure of the isolated monomer bisVBT, a by-product obtained from the VBT synthesis, was confirmed by proton NMR, IR, and X-ray crystal analysis. Moreover, three new synthesized materials were characterized by ${ }^{1}$ HNMR and GPC.

The crystal structure of bisVBT monomer and the unit cell are shown in Figures 2(a)-2(b), which reveals that the monomer molecules pack into tight layers. Nearby thymine moieties are generated by stacking of the layers, which brings the thymine groups slightly offset of the ring with an average thymine separation of $5.9 \AA$.

3.1. Gel Permeation Chromatography. The average molecular weights of the resultant bisVBT-VBA copolymers were measured by gel permeation chromatography (GPC) [27]. The primary limitation of conventional GPC is that the obtained molecular weights are relative values. Therefore, the accuracy of the method depends upon the standards and samples having the same relationship between their hydrodynamic volume, charge, and molecular weight. This is especially true for charged copolymers systems [20].
Given that bisVBT-VBA copolymers are positively charged, a poly(2-vinylpyridine) (PVP) was chosen as the standard, since it becomes positively charged upon exposure to an acidic mobile condition and exhibits similar interactions with the column phase as the cationic bisVBT-VBA copolymers. A calibration curve (log $\mathrm{Mw}$ versus retention volume) was created from PVP standard runs, which allows determination of copolymer molecular weight based on the retention volume. Table 1 shows the weight-average molecular weight (Mw), number-average molecular weight $(\mathrm{Mn})$, and polydispersity, $(\mathrm{Pd}=\mathrm{Mw} / \mathrm{Mn})$ of the bisVBT-VBA copolymers estimated from the calibration curves.

The results presented in Figure 3 show that all materials elute around 18 minutes, with average molecular weights ranging between 65,000 and $100,000 \mathrm{Da}$, and a possible secondary chain of copolymers with a slightly lower molecular weight. The differences in molecular weights for the bisVBTVBA structures are mainly due to variations in initiator concentration [8]. The lower amount AIBN used $(1: 32$ bisVBT-VBA 1\% AIBN) results in longer formed chains and therefore in higher molecular weight copolymers.

The fact that the terpolymer 1:4:32 bisVBT-VBT-VBA ( $1 \%$ AIBN) has the lowest molecular weight compared to bisVBT-VBA copolymers highlights a main difference between bisVBT and VBT monomers. The bisVBT monomer having two vinyl groups has higher quantity of sites to polymerize, giving rise to network structures and thereby increasing the molecular weight of the resulting materials as it is shown in Table 1. On the other side, the VBT monomer having only one vinyl group has lower possibilities to polymerize and generally will generate linear copolymers. Consequently, in the terpolymer $1: 4: 32$ bisVBT-VBT-VBA with a large amount of VBT monomer present, the expected molecular weight is lower than in bisVBT-VBA copolymers. From the GPC analysis it is likely that cross-linked network copolymers from bisVBT monomers will not be soluble, and therefore the observed GPC traces of filtered samples represent the soluble fraction of the materials with low degree of cross-linking. This might explain the unexpectedly low polydispersity (Pd) values of the copolymer molecular weights, compared to reported random VBT-VBA copolymerization reactions, which exhibit acceptable polydispersity values between 1.5 and $4[3,26,28,29]$.

3.2. Thermal Analysis. The thermal properties and stability of the bisVBT-VBA copolymer were studied with TGA in a nitrogen stream and with DSC. The TGA thermogravimetric curves of different bisVBT-VBA copolymers and the 1:8 VBT-VBA control copolymer are presented in Figure 4. For all copolymers, the initial 5 to $10 \%$ weight loss at $100^{\circ} \mathrm{C}$ occurs as a result of water evaporation. Figure 4(a) shows that both 1:32 bisVBT-VBA copolymers with $1 \%$ and $3 \%$ AIBN initiator have similar features, indicating that the small difference in MW has no influence on the thermal properties. The thermograms have two very well-defined degradation steps. The first step of $40 \%$ weight loss occurring at $209^{\circ} \mathrm{C}$ corresponds to VBA monomer. The second degradation point for both 1:32 bisVBT-VBA materials corresponds to bisVBT monomer and was found at $445^{\circ} \mathrm{C}$ (Figure $4(\mathrm{a})$ ). This value 


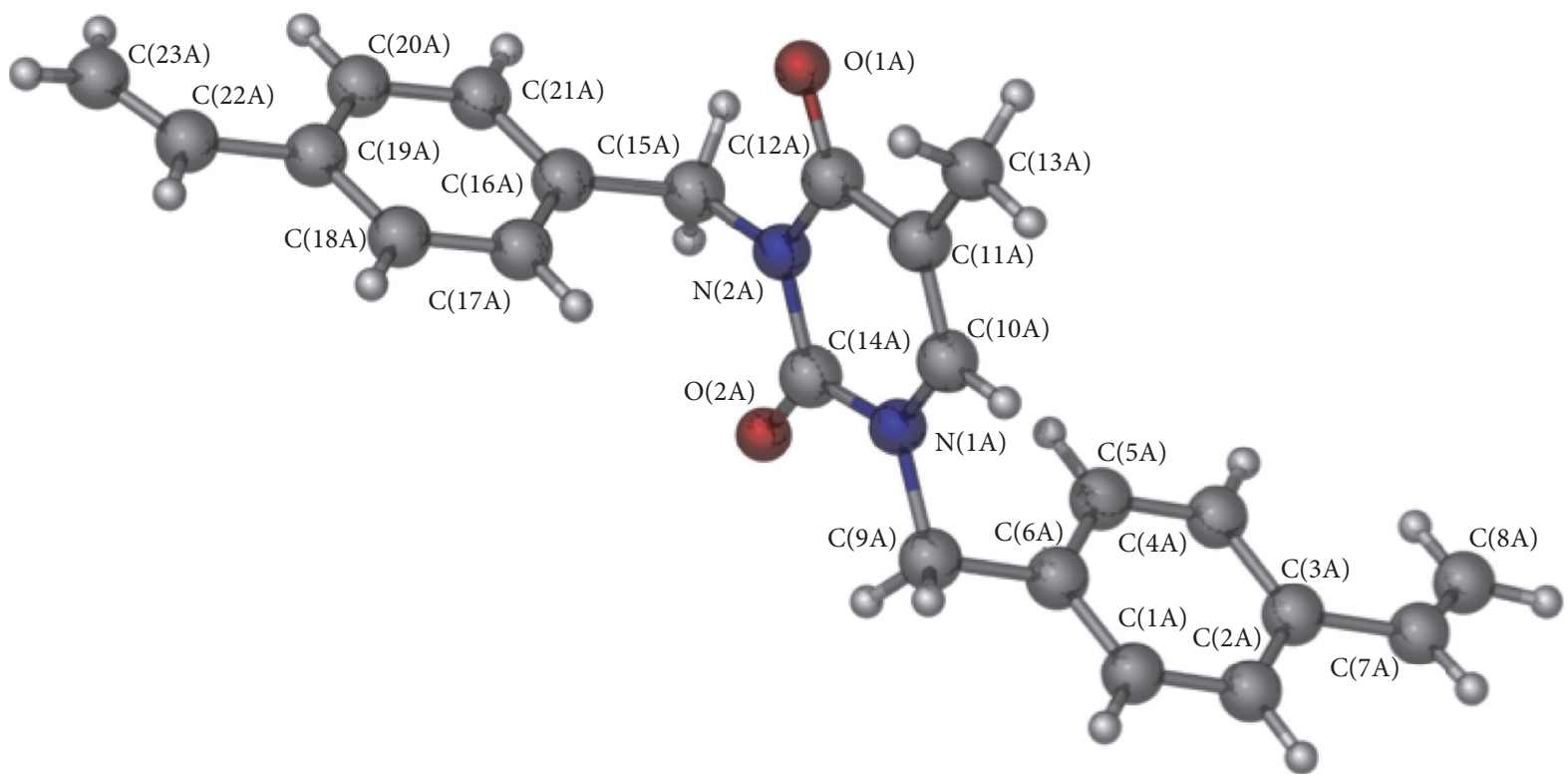

(a)

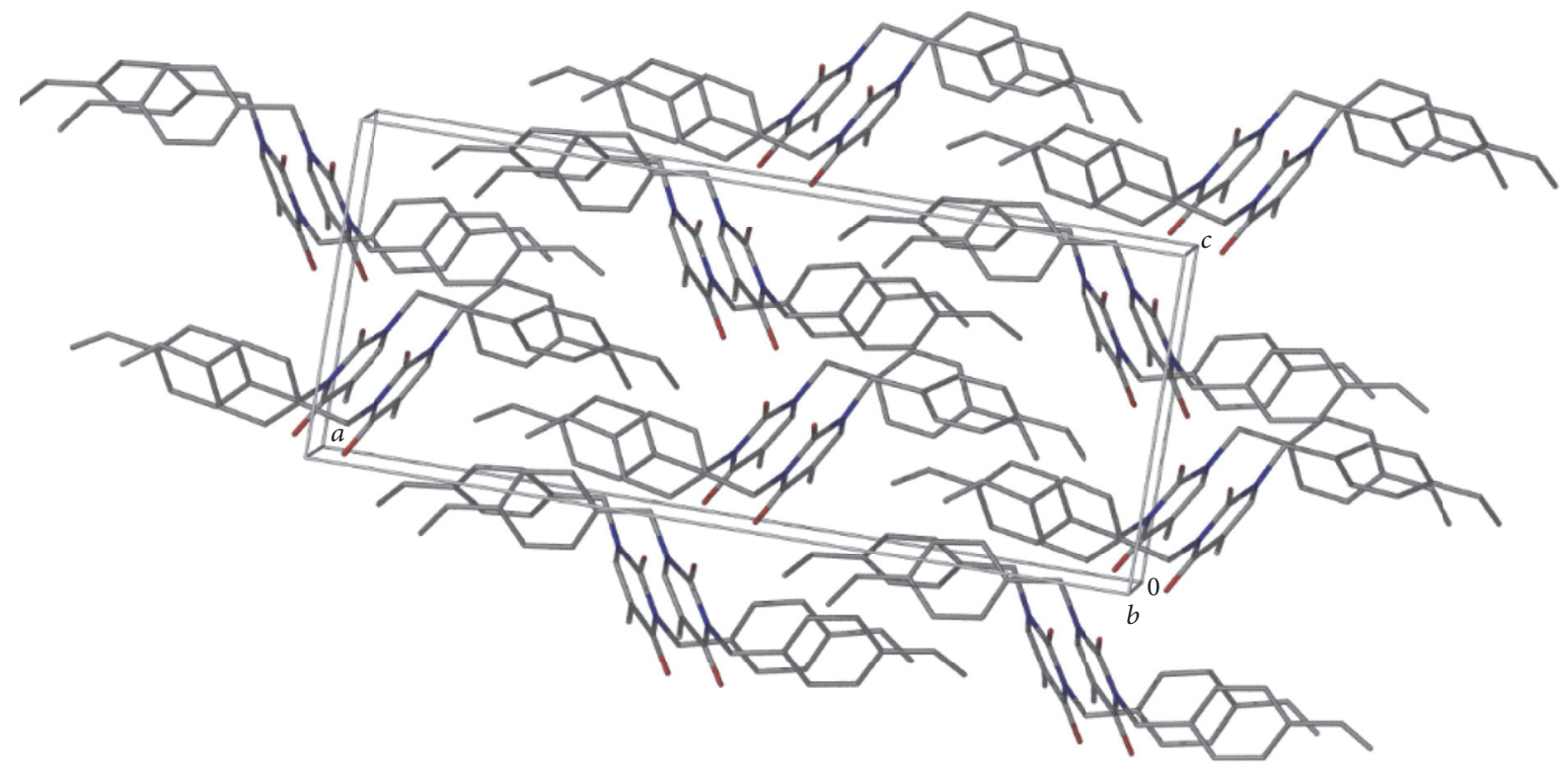

(b)

FIGURE 2: (a) The bisVBT monomer crystal structure with unique carbons labeled and (b) bisVBT monomer alignment in the unit cell.

TABLE 1: Average molecular weights of bisVBT-VBA samples determined by GPC, using poly(2-vinylpyridine) calibration curves.

\begin{tabular}{|c|c|c|c|c|}
\hline Copolymer sample & $\begin{array}{l}\text { Retention time } \\
(\min )\end{array}$ & $\mathrm{Mw}(\mathrm{Da})$ & $\mathrm{Mn}$ & $\mathrm{Pd}$ \\
\hline $\begin{array}{l}1: 32 \text { bisVBT-VBA } \\
(1 \% \text { AIBN })\end{array}$ & 18.003 & 100,920 & 89,730 & 1.12 \\
\hline $\begin{array}{l}1: 32 \text { bisVBT-VBA } \\
(3 \% \text { AIBN })\end{array}$ & 18.098 & 90,720 & 80,270 & 1.12 \\
\hline $\begin{array}{l}1: 4: 32 \text { bisVBT-VBT-VBA } \\
(1 \% \text { AIBN })\end{array}$ & 18.412 & 64,020 & 56,900 & 1.13 \\
\hline
\end{tabular}




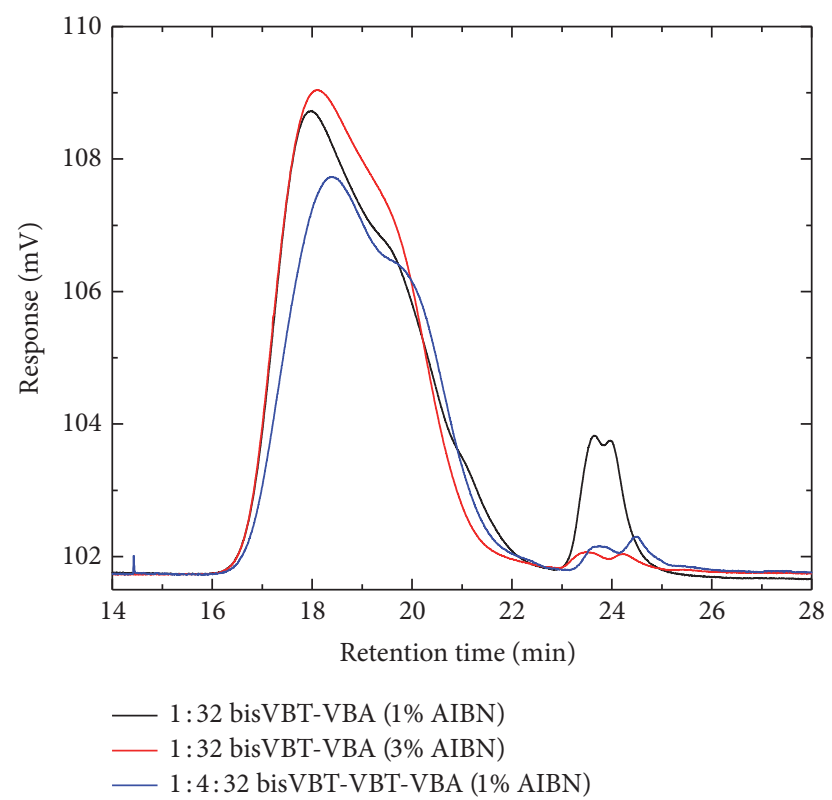

Figure 3: Overlaid GPC chromatograms of three bisVBT-VBA samples.

is slightly higher than the VBT degradation presented for the $1: 8$ VBT-VBA copolymer $\left(390^{\circ} \mathrm{C}\right)$ and the $1: 4: 32$ bisVBTVBT-VBA terpolymer $\left(400^{\circ} \mathrm{C}\right.$ ) (as shown in Figure $4(\mathrm{~b})$ ).

While the VBA monomer ratio decreases going from $1: 32$ bisVBT-VBA to 1:8 VBT-VBA copolymers, the first degradation step related to VBA monomer takes place at a lower temperature, as it can be observed for 1:8 VBT-VBA (1\% AIBN) copolymer at $195^{\circ} \mathrm{C}$ (Figure 4(b)). These observations suggest that bisVBT-VBA copolymers have greater thermal flexibility than VBT-VBA copolymers.

An important information when evaluating new materials for their potential application is the glass transition temperature (Tg), which is defined as a change in heat capacity when the polymer matrix changes from "glass" at low temperatures to "rubber" at higher temperatures. This second-order endothermic transition appears in the thermogram as a step not a peak. From DSC thermograms (not shown) no distinguishable thermal phase transitions were observed, besides slight steps from where the Tg for each material was determined.

The DSC thermograms showed a decrease in Tg values when the VBA ratio in the copolymer increases going from $89.3^{\circ} \mathrm{C}$ for 1 : 8 VBT-VBA copolymers to approximately $74.5^{\circ} \mathrm{C}$ for 1:32 bisVBT-VBA copolymers. This can be explained since a higher content of cationic VBA monomers increases electrostatic repulsions between monomers, which makes the material less rigid. When comparing materials having similar VBT : VBA ratio, it can be observed that for $1: 8 \mathrm{VBT}-$ VBA copolymers the Tg value $\left(89.3^{\circ} \mathrm{C}\right)$ is higher than for the $1: 4: 32$ bisVBT-VBT-VBA terpolymer $\left(76^{\circ} \mathrm{C}\right)$ indicating that the presence of bisVBT monomer may act as a "plasticizer," which improves the fluidity and reduces the brittleness of the material. In general, the presence of high $\mathrm{Tg}$ values was expected considering that the synthesized structures have high molecular weights.

The glass transition temperatures ( $\mathrm{Tg}$ ) of the bisVBTVBA structures are lower than those of the VBT-VBA copolymers, supporting the previous conclusion that arrangements containing the bisVBT monomer have greater thermal flexibility than those containing the VBT monomer.

3.3. Qualitative Irradiation Studies. All three bisVBT-VBA synthesized materials and the control 1: 8 VBT-VBA copolymer were layered on thin films and irradiated for different times $30 \mathrm{sec}, 60 \mathrm{sec}, 120 \mathrm{sec}$, and $180 \mathrm{sec}$ (equivalent to the amount of energy delivered). The VBT films are colorless and in order to quantify the extent of their photoimmobilization on the substrate, the films were toned with an anionic FD\&C Blue dye. The dye molecules have low affinity to the PET substrate, nevertheless strongly interacting with the immobilized oppositely charged polycationic copolymer. Thus, the thymine cross-linking can be indirectly estimated by analyzing the dye adsorption onto the insoluble copolymer.

The immobilization degree between the substrate and the copolymer is affected by the molar mass and the chemical composition. At shorter times the photo cross-linking and the resulting increase in average molecular weight of the copolymers do not affect solubility. Nevertheless, as the reaction proceeds and more dimers are generated, the copolymer chains form high molecular weight networks, which make up the insoluble fraction of the photo-cross-linked copolymer. When all copolymer chains are connected a saturation level is reached, and continued irradiation does not cause any additional decrease in solubility. This is a typical behavior of photoresist materials [30].

Figure 5 shows qualitatively how the extent of immobilization varies between all bisVBT-VBA cross-linked materials. In all cases it was observed that the amount of trapped dye increases with irradiation time until it reaches saturation (time evolution not shown). Out of the three new synthetic copolymers, the 1:4:32 bisVBT-VBT-VBA (1\% AIBN) terpolymer in 50/50 MeOH-water (Figure 5(a)) exhibited a satisfactory degree of cross-linking, as all the photo-masked area remained immobilized. As a comparison, the previously studied copolymer 1: 8 VBT-VBA (1\% AIBN) [31-33] in 50/50 $\mathrm{MeOH}$-water was used as a control (Figure 5(b)). Visually, the photo cross-linking exhibited by both copolymers was comparable, as it was expected since they have similar VBTVBA ratios.

A moderate degree of cross-linking occurred with the 1:32 bisVBT-VBA (3\% AIBN) copolymer in 50/50 MeOHwater (Figure 5(c)), where it can be observed that longer irradiation times $(120 \mathrm{sec})$ were needed to immobilize copolymer. These circumstances were expected for the reason that, when less fraction of cross-linkable VBT compared with VBA monomer is present in the copolymer films, few thymine groups undergo photodimerization, and therefore longer irradiation time is required for immobilization. Therefore, decreasing bisVBT/VBT concentration will result in thymine molecules further away from each other, and in consequence a slower and less efficient photo cross-link. In summary, copolymers with high VBA content will possess a decreased 


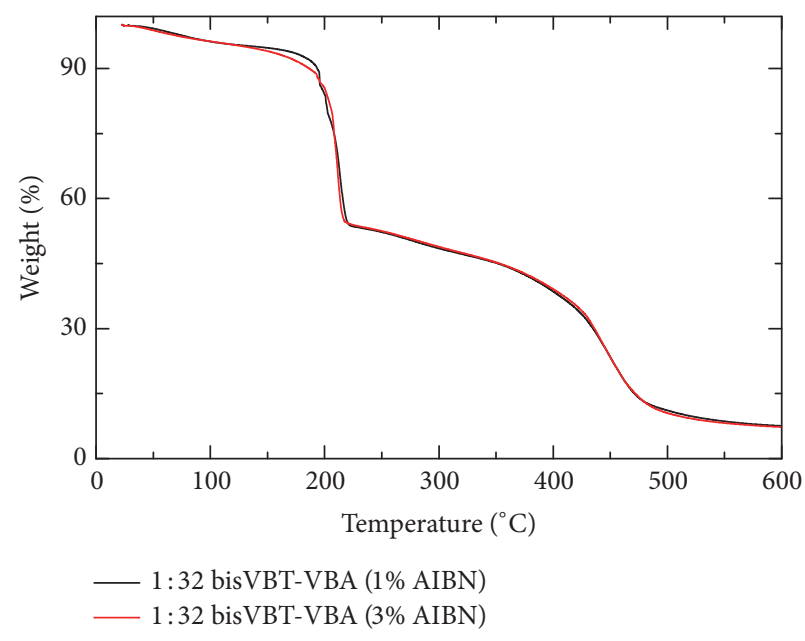

(a)

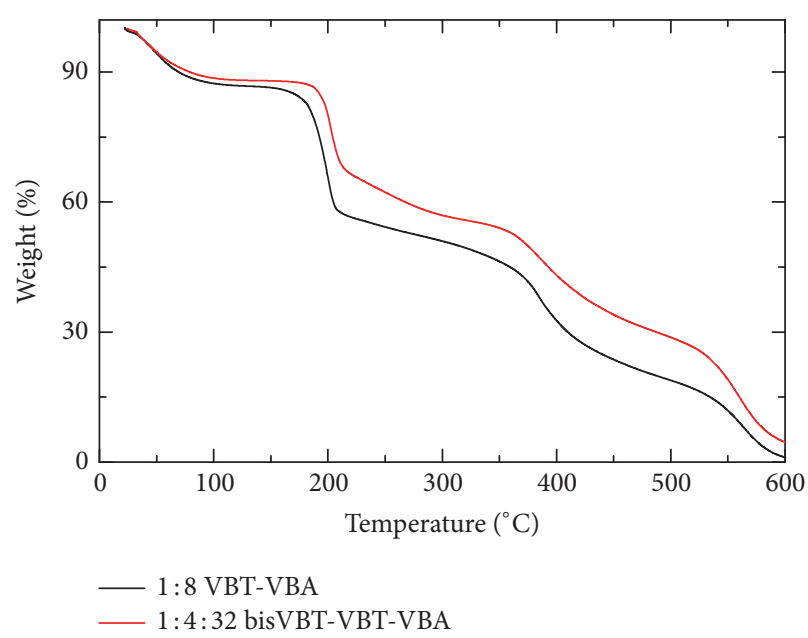

(b)

FIGURE 4: TGA thermograms of the copolymer systems under nitrogen atmosphere: (a) 1:32 bisVBT-VBA 1\% AIBN (black line) and 3\% AIBN (red line); (b) 1:8 VBT-VBA (black line) and 1:4:32 bisVBT-VBT-VBA (red line).

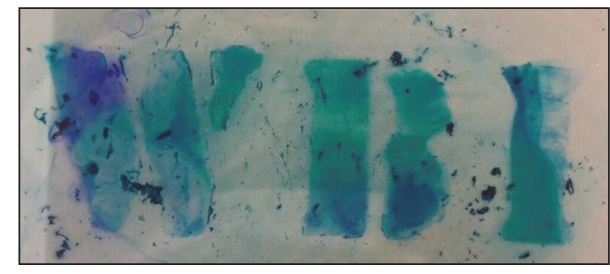

(a)

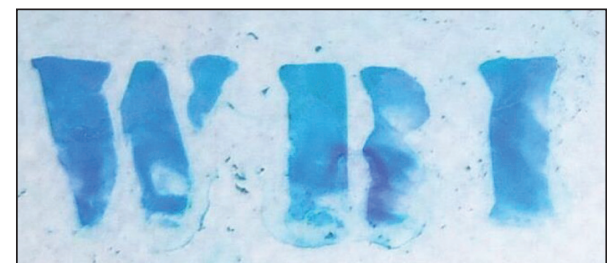

(b)

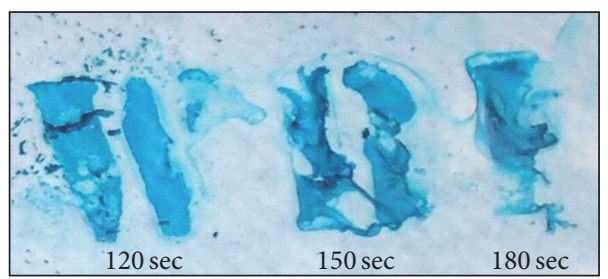

(c)

Figure 5: (a) 1:4:32 bisVBT-VBT-VBA (1\% AIBN) structure irradiated for $60 \mathrm{sec}$; (b) 1:8 VBT-VBA (1\% AIBN) copolymer irradiated for $60 \mathrm{sec}$; (c) 1:32 bisVBT-VBA ( $3 \%$ AIBN) structure irradiated for $120 \mathrm{sec}, 150 \mathrm{sec}$, and $180 \mathrm{sec}$. All copolymers were 10\% solutions in $50 / 50$ $\mathrm{MeOH} /$ water.

ability to cross-link when irradiated with UV light mostly due to a low content of VBT monomers, but correspondingly as a result of increased electrostatic repulsions between VBA monomers (low $\mathrm{Tg}$ ) which hinders the free spatial arrangement of the copolymer reducing the possibility of adjacent thymine moieties to be close enough to cross-link. Figure 5(c) also shows that bisVBT-VBA structures have a typical photoresist behavior, since after 120 sec of irradiation the dye adsorption reaches saturation.

While the intention of increasing the molar equivalence of VBA to bisVBT was to increase the solubility of the bisVBT-VBA materials, the smaller molar equivalence of bisVBT may have caused the copolymer chains to have insufficient amounts of neighboring thymine moieties in the proximity, required for photodimerization and adequate immobilization. Quantitative studies of the photo cross-linking of copolymers varying bisVBT to VBA ratios are currently under progress.

It is worthy to note that the bisVBT-VBA copolymers in a $10 \% \mathrm{w} / \mathrm{w}$ solution in $50 / 50 \mathrm{MeOH}$-water exhibited a tendency to swell upon solvation in hydroxyl containing solvents, like water and alcohols. This property is consistent with crosslinked copolymers and may reduce the processability of the new materials, increasing the difficulty of creating uniform thickness films.

\section{Conclusions}

The VBT and bisVBT monomers, in addition to specifically formulated copolymers/network structures, were successfully synthesized and characterized. The identities of the VBT and the novel bisVBT monomers were confirmed via ${ }^{1} \mathrm{H}$ 
NMR, ${ }^{13} \mathrm{C}$ NMR, IR, and X-ray crystallography. Currently, a design of experiments to study the conditions to maximize the yield of either VBT or bisVBT monomers is underway and will be reported in the future.

Specifically formulated copolymers/network structures of bisVBT, VBT, and VBA monomers were synthesized. Qualitative irradiation studies of the synthesized copolymers/network structures revealed that, to some extent, an immobilized film can be generated. The irradiation studies also revealed that the synthesized structures containing bisVBT exhibited a significant level of swelling upon solvation with hydroxyl containing solvents (water and polar solvents). The swelling property causes the viscosity of the copolymers to increase tremendously and should be an important consideration in characterization as well as in applications design.

The production and isolation of the bisVBT monomer introduce numerous interesting areas to explore in the future. The synthesis of copolymers with increased ratios of bisVBT to VBA monomers is needed to evaluate the mechanical properties of the immobilized films. The particle size distribution of the resulting copolymers using Dynamic Light Scattering (DSL) would provide an insight into potential nanogel formation. Additional comparative swelling and solubility studies need to be undertaken to quantify the amount of cross-linking on structures with different bisVBT-VBA ratios. These complementary studies and a further knowledge of the bisVBT monomer properties will potentially move forward to the development of valuable cross-linked copolymers.

\section{Competing Interests}

The authors declare that there is no conflict of interests regarding the publication of this paper.

\section{Acknowledgments}

Authors would like to thank the William M. Keck Foundation for the support to this project. Thanks are due to Bill Dorogy for providing the $1: 8 \mathrm{VBT}-\mathrm{VBA}$ copolymer.

\section{References}

[1] J.-F. Lutz, A. F. Thünemann, and R. Nehring, "Preparation by controlled radical polymerization and self-assembly via base-recognition of synthetic polymers bearing complementary nucleobases," Journal of Polymer Science, Part A: Polymer Chemistry, vol. 43, no. 20, pp. 4805-4818, 2005.

[2] Y. Imai, T. Ogoshi, K. Naka, and Y. Chujo, "Formation of IPN organic-inorganic polymer hybrids utilizing the photodimerization of thymine," Polymer Bulletin, vol. 45, no. 1, pp. 9-16, 2000.

[3] Y. Dahman, J. E. Puskas, A. Margaritis, Z. Merali, and M. Cunningham, "Novel thymine-functionalized polystyrenes for applications in biotechnology. Polymer synthesis and characterization," Macromolecules, vol. 36, no. 7, pp. 2198-2205, 2003.

[4] H. Xu, R. Hong, T. Lu, O. Uzun, and V. M. Rotello, "Recognition-directed orthogonal self-assembly of polymers and nanoparticles on patterned surfaces," Journal of the American Chemical Society, vol. 128, no. 10, pp. 3162-3163, 2006.

[5] C. M. Cheng, M. I. Egbe, J. M. Grasshoff et al., "Synthesis of 1-(vinylbenzyl)thymine, a novel, versatile multi-functional monomer," Journal of Polymer Science Part A: Polymer Chemistry, vol. 33, no. 14, pp. 2515-2519, 1995.

[6] X. Yang, P. Xu, S. Ding, M. Radosz, and Y. Shen, "Synthesis of 1-(4-vinylbenzyl)thymine and its polymerization by RAFT and ATRP techniques," Polymer Preprints, vol. 45, no. 1, pp. 10611062, 2004.

[7] C. Kiarie, J. Bianchini, S. Trakhtenberg, and J. C. Warner, "Methylene blue adsorption on thymine based polyvinylphenylsulfonate films," Journal of Macromolecular Science, Part A: Pure and Applied Chemistry, vol. 42, no. 11, pp. 1489-1496, 2005.

[8] A. L. Barbarini, D. A. Estenoz, and D. M. Martino, "Synthesis, characterization and curing of bioinspired polymers based on vinyl benzyl thymine and triethyl ammonium chloride," Macromolecular Reaction Engineering, vol. 4, no. 6-7, pp. 453459, 2010.

[9] D. M. Martino, D. Reyna, D. A. Estenoz, S. Trakhtenberg, and J. C. Warner, "Photosensitization of bioinspired thyminecontaining polymers," Journal of Physical Chemistry A, vol. 112, no. 21, pp. 4786-4792, 2008.

[10] A. L. Barbarini, D. L. Reyna, and D. M. Martino, "The effect of light intensity, film thickness, and monomer composition in styrene-based bioinspired polymers," Green Chemistry Letters and Reviews, vol. 3, no. 3, pp. 231-237, 2010.

[11] J. M. Grasshoff, L. D. Taylor, and J. C. Warner, U.S. Patent 5,455,349, October 1995.

[12] J. M. Grasshoff, L. D. Taylor, and J. C. Warner, "Method of imaging using a polymeric photoresist having pendant vinylbenzyl thymine groups," US Patent 5,616,451, April 1997.

[13] J. M. Grasshoff, L. D. Taylor, and J. C. Warner, US Patent 5,708,106, January 1998.

[14] J. C. Warner, A. S. Cannon, J. Raudys, and A. Undurti, PCT International Application WO 2004058187, December 2004.

[15] Y. Inaki, "Thymine polymers as high resolution photoresists and reversiblephoto-recording materials," Polymer News, vol. 17, pp. 367-371, 1992.

[16] S. Trakhtenberg, J. C. Warner, R. Nagarajan, F. F. Bruno, L. A. Samuelson, and J. Kumar, "Spectroscopic and microscopic analysis of photo-cross-linked vinylbenzylthymine copolymers for photoresist applications," Chemistry of Materials, vol. 18, no. 12, pp. 2873-2878, 2006.

[17] R. F. El-Hayek, K. Dye, and J. C. Warner, "Bacteriostatic polymer film immobilization," Journal of Biomedical Materials Research Part A, vol. 79, no. 4, pp. 874-881, 2006.

[18] A. L. Barbarini, D. A. Estenoz, and D. M. Martino, "Crosslinkable micelles from diblock amphiphilic copolymers based on vinylbenzyl thymine and vinylbenzyl triethylammonium chloride," Journal of Applied Polymer Science, vol. 132, no. 19, Article ID 41947, 2015.

[19] K. Saito and J. C. Warner, "Core-shell thymine containing polymeric micelle system: study of controlled release of riboflavin," Green Chemistry Letters and Reviews, vol. 2, no. 2, pp. 71-76, 2009.

[20] G. Kaur, S. L. Y. Chang, T. D. M. Bell, M. T. W. Hearn, and K. Saito, "Bioinspired core-crosslinked micelles from thyminefunctionalized amphiphilic block copolymers: Hydrogen Bonding and Photo-crosslinking Study," Journal of Polymer Science, Part A: Polymer Chemistry, vol. 49, no. 19, pp. 4121-4128, 2011. 
[21] J. R. Whitfield, A. Morelli, and J. C. Warner, "Enzymatic reversal of polymeric thymine photocrosslinking with E. coli DNA photolyase," Journal of Macromolecular Science Part A: Pure and Applied Chemistry, vol. 42, no. 11, pp. 1541-1546, 2005.

[22] V. I. Paz Zanini, F. Tulli, D. M. Martino, B. López de Mishima, and C. D. Borsarelli, "Improvement of the amperometric response to L-lactate by using a cationic bioinspired thymine polycation in a bioelectrode with immobilized lactate oxidase," Sensors and Actuators B: Chemical, vol. 181, pp. 251-258, 2013.

[23] V. I. Paz Zanini, M. Gavilán, B. A. López De Mishima, D. M. Martino, and C. D. Borsarelli, "A highly sensitive and stable glucose biosensor using thymine-based polycations into laponite hydrogel films," Talanta, vol. 150, pp. 646-654, 2016.

[24] P. T. Anastas and J. C. Warner, Green Chemistry: Theory and Practice, Oxford University Press, Oxford, UK, 1998.

[25] P. Zarras and O. Vogl, "Polycationic salts. 3. synthesis, styrene based trialkylammonium salts and their polymerizationl-2," Journal of Macromolecular Science A, vol. 37, no. 8, pp. 817-840, 2000.

[26] J. R. Bianchini, K. Saito, T. B. Balin, V. Dua, and J. C. Warner, "Thymine-based, water-soluble phototerpolymers: their preparation and synthesis," Journal of Polymer Science, Part A: Polymer Chemistry, vol. 45, no. 7, pp. 1296-1303, 2007.

[27] A. L. McLaughlin, Development of a method for characterizing synthetic polymer simples using gel permeation chromatography and poly-vinylbenzyl chloride standard curve [B.S. thesis], Simmons College, Boston, Mass, USA, 2012.

[28] S. A. Bortolato, A. L. Barbarini, R. M. Benitez, D. A. Estenoz, and D. M. Martino, "Photo-induced curing of thymine-based bioinspired polymers. A chemometric analysis," Latin American Applied Research, vol. 43, pp. 329-336, 2013.

[29] S.-W. Kuo and R.-S. Cheng, "DNA-like interactions enhance the miscibility of supramolecular polymer blends," Polymer, vol. 50, no. 1, pp. 177-188, 2009.

[30] A. Reiser and E. A. Pitts, "A photographic theory of crosslinking resists," The Journal of Photographic Science, vol. 29, no. 5, pp. 187-191, 1981.

[31] S. Trakhtenberg, A. S. Cannon, and J. C. Warner, "Chapter 13 non-catalytic photo-induced immobilization processes in polymer films," Thin Films and Nanostructures, vol. 34, pp. 665695, 2007.

[32] J. Ledesma, S. A. Bortolato, C. E. Boschetti, and D. M. Martino, "Optimization of environmentally benign polymers based on thymine and polyvinyl sulfonate using plackett-burman design and surface response," Journal of Chemistry, vol. 2013, Article ID 947137, 9 pages, 2013.

[33] L. O. Searls, Synthesis and characterization of thymine-based photoresist polymers [B.S. thesis], Simmons College, Boston, Mass, USA, 2009. 

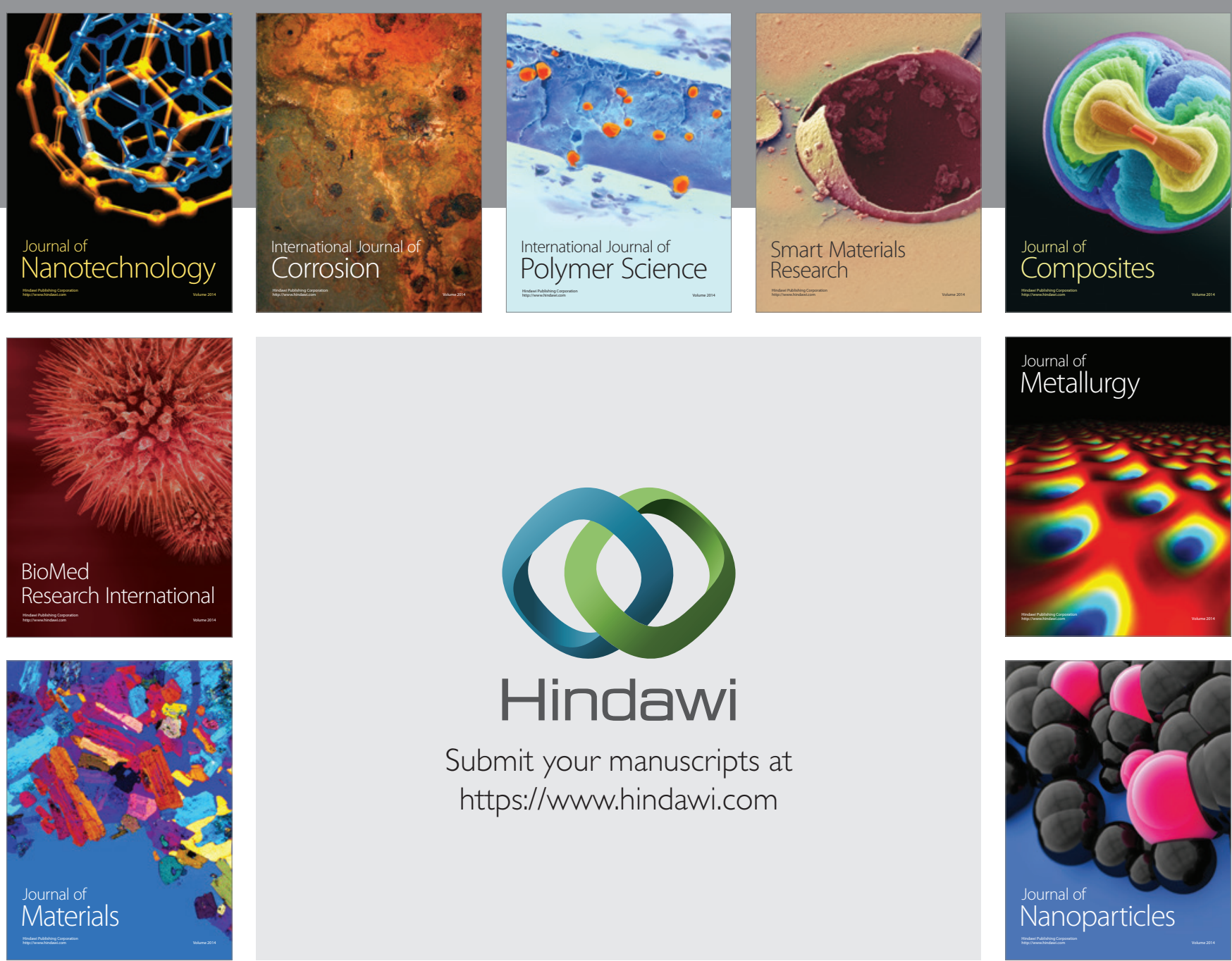

\section{Hindawi}

Submit your manuscripts at

https://www.hindawi.com

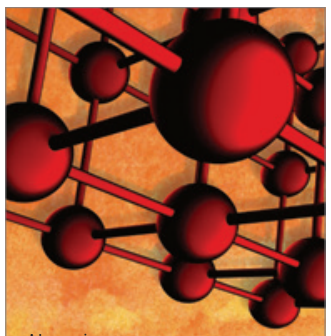

Materials Science and Engineering
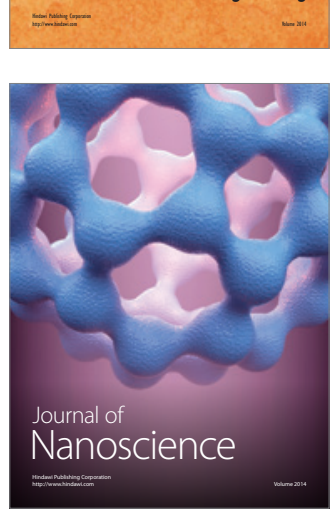
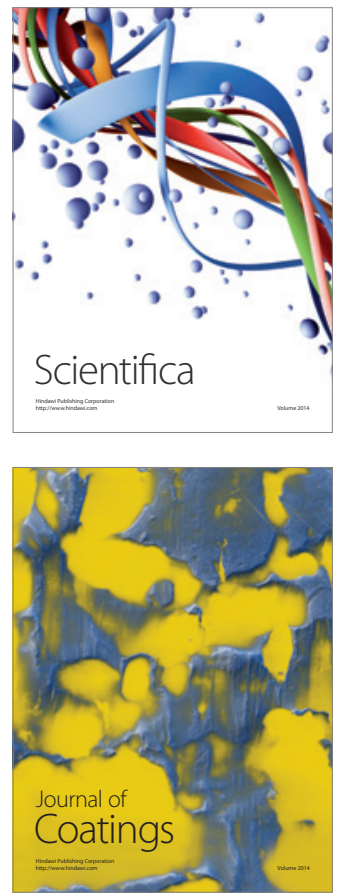
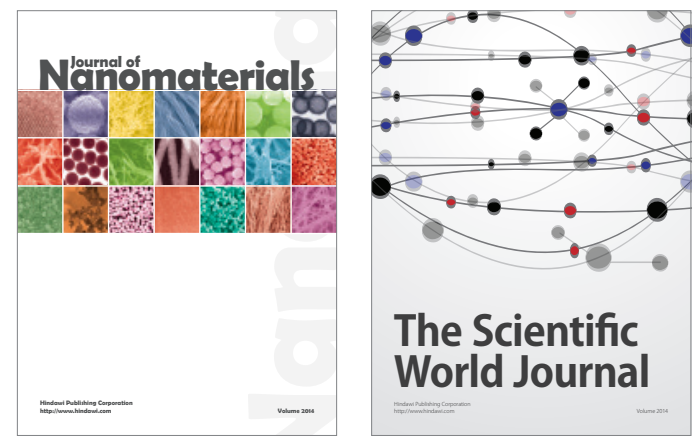

The Scientific World Journal
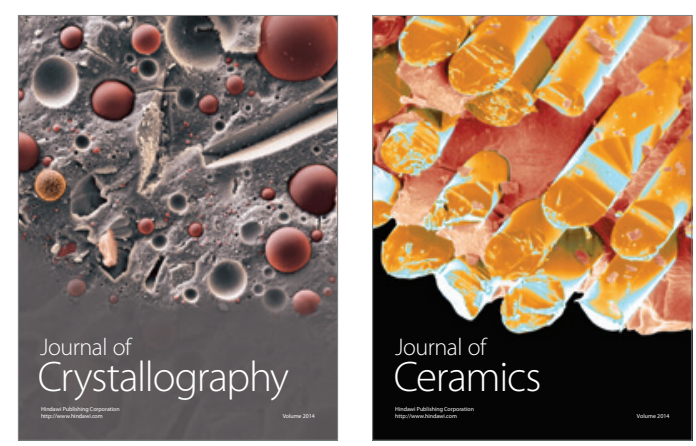
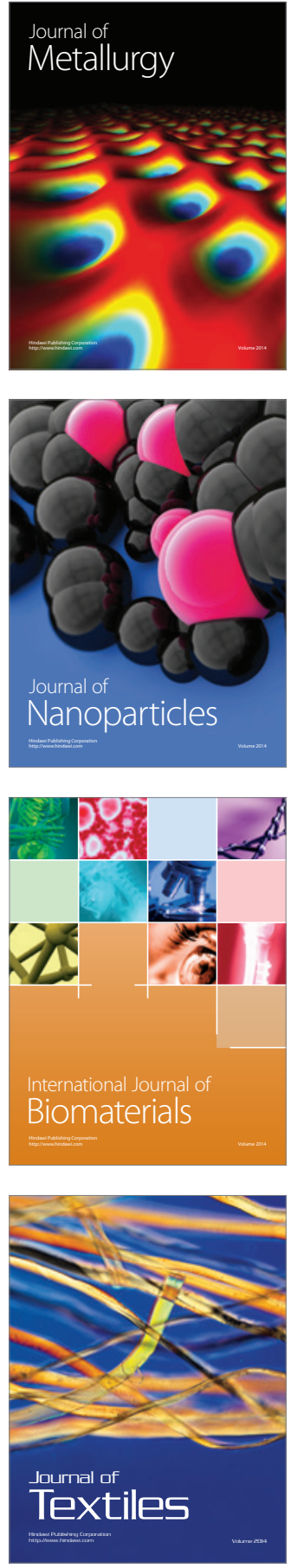